Journal of Electronics and Informatics (2020)

Vol.02/ No. 01

Pages: 49-71

http://www.irojournals.com/iroei/

DOI: https://doi.org/10.36548/jei.2020.1.006

\title{
EL DAPP - An Electrıcıty Meter Trackıng Decentralızed Applıcatıon
}

Dr. N. Bhalaji,

Department of Information Technology,

SSN College of Engineering, Kalavakkam,

Chennai, TamilNadu, India.

Email id:bhalajin@ssn.edu.in

Shanmuga Skandh Vinayak E

Department of Information Technology,

SSN College of Engineering, Kalavakkam,

Chennai, TamilNadu, India.

Email id:shanmugaskandhvinayak16095@it.ssn.edu.in

\begin{abstract}
The electricity industry has always been under scrutiny in order to improve the quality of electricity supply, measurement and billing services to have the at most user transparency, while providing these services with the highest efficiency. Although many solutions have emerged, of which the smart meter was considered a viable option, it was quick to perish under the prodigious complications with the real-life feasibilities. El DApp - An electricity power consumption tracking application solution, harnessing both the IoT and Blockchain utilities to provide a decentralized and secure recording mechanism, that provides an improved architecture to the smart meter is proposed in this article. The El DApp provides a high security and cost efficient decentralized live electricity power consumption recording of the user that is maintained by a Raspberry Pi based Ethereum network.
\end{abstract}

Keywords - El DApp, Decentralized Application, energy meter, blockchain, IoT, Raspberry Pi, Ethereum

\section{Introduction}

The electricity sector has been an ever-growing industry, as majority of the economies are adapting industrialization. The global power consumption is at its peak in the current state consuming over 4000 thousand units of power per capita [1]. This market pave way to smart solutions that efficiently, accurately and securely measure the usage with minimum man power. 
Journal of Electronics and Informatics (2020)

Vol.02/ No. 01

Pages: 49-71

http://www.irojournals.com/iroei/

DOI: https://doi.org/10.36548/jei.2020.1.006

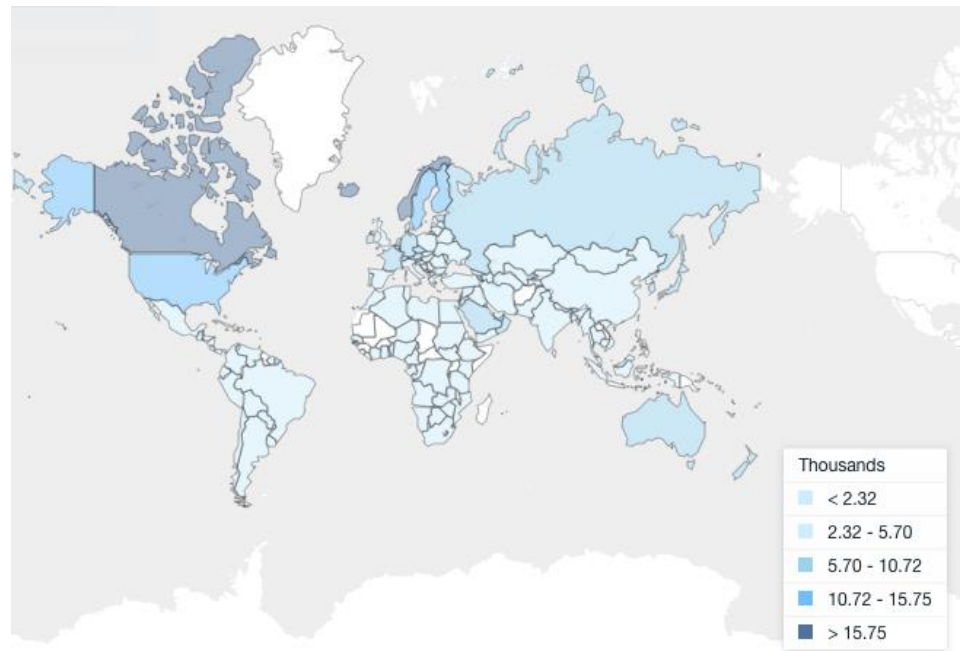

Fig. 1. Electric power consumption (kWh per capita, country-wise)

The conventional method of measuring electricity, majorly adapted by most of the regions utilizing domestic electricity are the variations of the mechanical meter that requires extensive manual labor in measuring and generating electricity bills for every site user [figure 2].

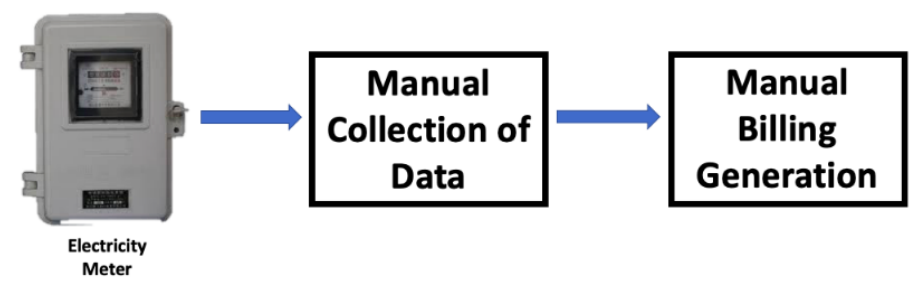

Fig. 2. Conventional Energy Meter Architecture

One of the major solutions in improving the conventional energy meter system is the smart energy meter [Fig. 3]. The smart meter takes the advantages of the client server architecture in providing a modernized solution to the man power inducing energy meter while also providing an architecture that can measure the electricity accurately.

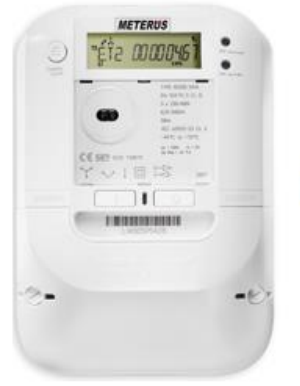

Electricity

Meter

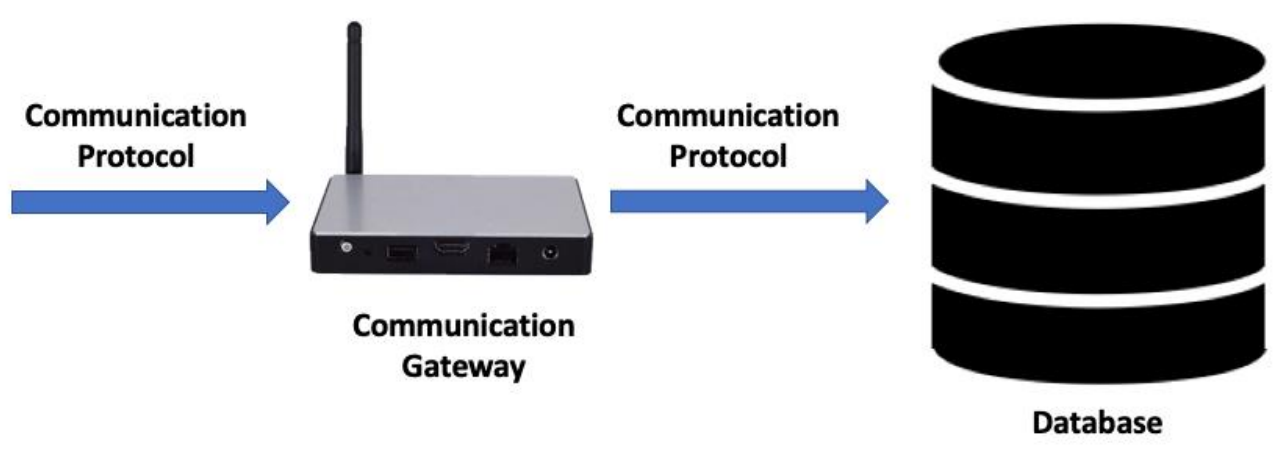

ISSN: 2582-3825 (online) 
Journal of Electronics and Informatics (2020)

Vol.02/ No. 01

Pages: 49-71

http://www.irojournals.com/iroei/

DOI: https://doi.org/10.36548/jei.2020.1.006

Fig. 3. Smart Energy Meter Architecture

While the solution may reduce manual labor in measuring energy consumption accurately, some of the major drawbacks are as follows.

- Internet resources that educates the masses on techniques to hack into the meter, tampering with the readings that places the individuals at a liability issue [14,15].

- The unreliability of the smart meter [16].

- The cost of centralization of the user records that includes storage, maintenance and security.

- The problem of a single point of failure that follows a centralized architecture.

These problems are addressed in the proposed model.

\section{Proposed Model}

In this article a solution combing the competencies of the IoT and blockchain technologies, to smartly measure the electricity power consumption in the domestic sector. The $21^{\text {st }}$ century is one of the most pivotal periods of time that utilizes smart devices adapting to integrating with automation solutions to improve the quality of services provided to consumers. Amongst such solutions, the IoT technologies emulate majority of the smart solutions by being able to provide inter-networked solutions. According to Statista, the number of connected devices installed base worldwide will reach up to 42.62 billion by the year 2022 and to about 75.44 by the year 2025 [2] with a revenue of 3 billion US dollars sectioned globally for the IoT security solutions by the year 2021 [3].

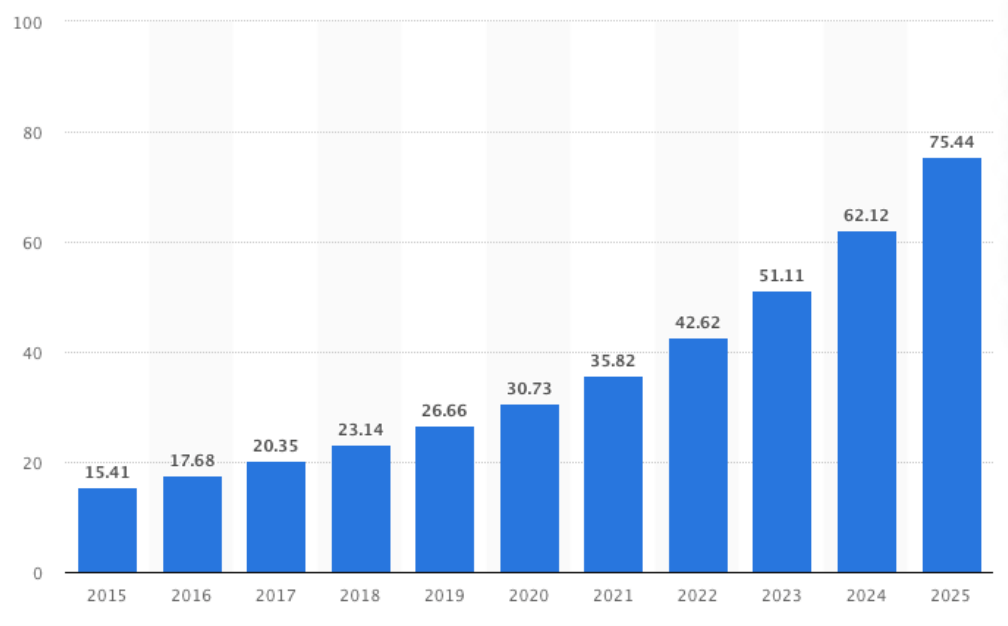

Fig. 4. Connected IoT devices world-wide

ISSN: 2582-3825 (online) 
Journal of Electronics and Informatics (2020)

Vol.02/ No. 01

Pages: 49-71

http://www.irojournals.com/iroei/

DOI: https://doi.org/10.36548/jei.2020.1.006

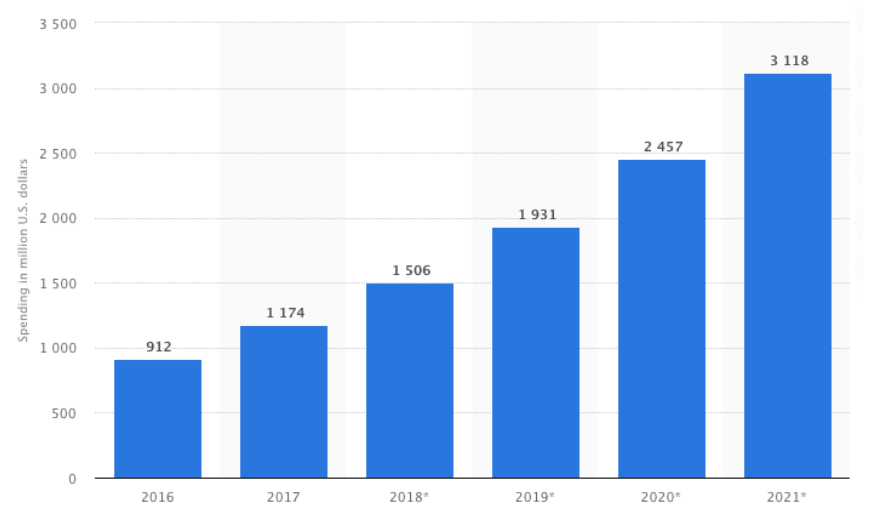

Fig. 5. Global spending for IoT security solutions

Figure 6 shows the statistics on the market revenue for the adaption and implementation of IoT solutions in energy meters in the United States [4].

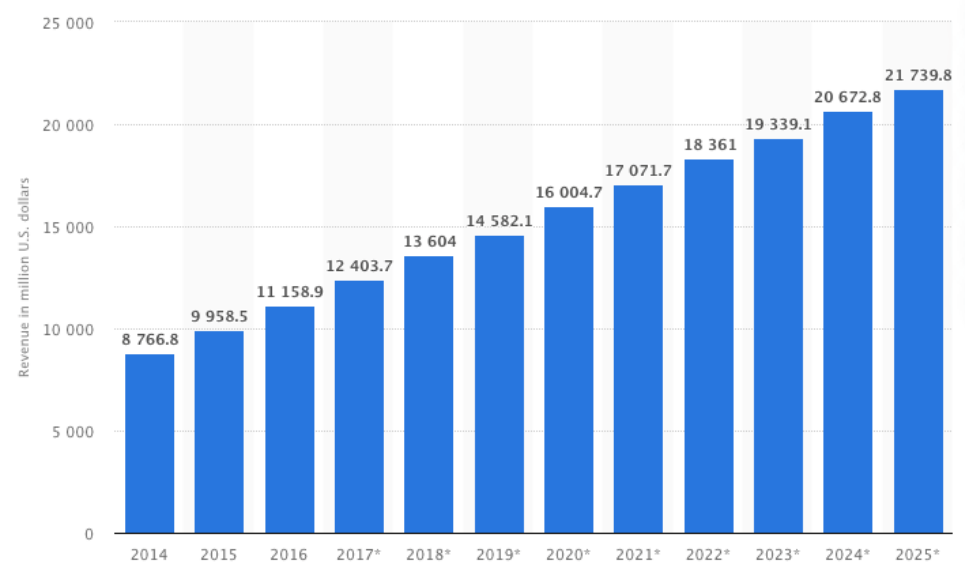

Fig. 6. Smart energy meter market revenue in the United States

These statistics show the direction in which the automation and the smart solutions market is moving toward technological advancements by adapting IoT solutions.

According to Statista, Blockchain is one of the most popularly emerging technologies, as it is being used as a viable solution in commercial solutions in both public and private sector to implement integrity and security [5]. Figure 7. shows the statistics of the blockchain impact on commercial sector. 
Journal of Electronics and Informatics (2020)

Vol.02/ No. 01

Pages: 49-71

http://www.irojournals.com/iroei/

DOI: https://doi.org/10.36548/jei.2020.1.006

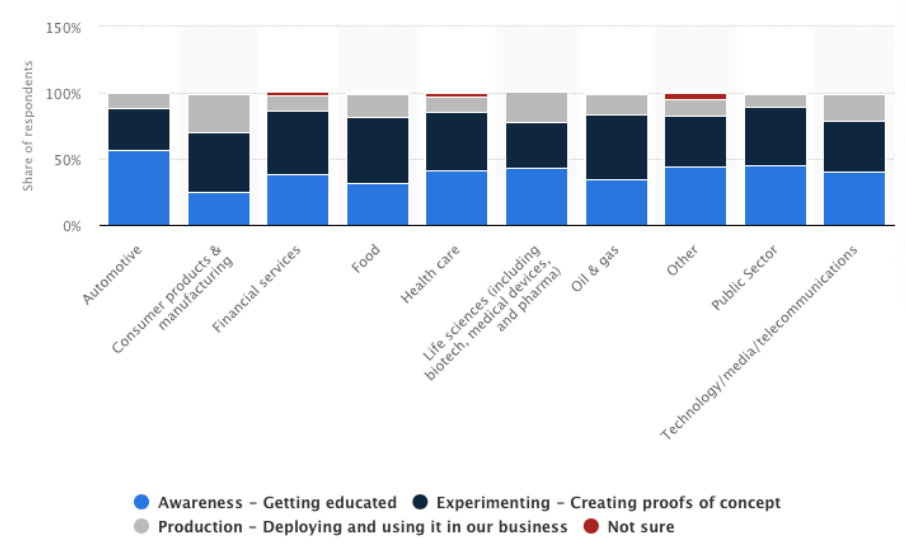

Fig. 7. Blockchain impact in Commercial Sector

The statistics show us that blockchain is being treated as a viable option to keep track of the transactions in a secure decentralized manner also opens a path for a collaboration of the IoT and blockchain technologies to provide a cheap and secure connected infrastructure in the business solutions. Blockchains are being adopted as a trusted solution while being integrated in architectural framework of several private companies to be tested for a use case that could improve the services provided by them. Through the utilization of blockchain to secure the IoT network, the drawbacks of a traditional client server architecture with respect to security can be addressed [17].

The architecture of the proposed model [Fig. 8] utilizes the IoT technology to host the application, hence rendering a server-less environment along with the blockchain technology to attain data decentralization thereby circumventing the single point of failure problem. 
Journal of Electronics and Informatics (2020)

Vol.02/ No. 01

Pages: 49-71

http://www.irojournals.com/iroei/

DOI: https://doi.org/10.36548/jei.2020.1.006

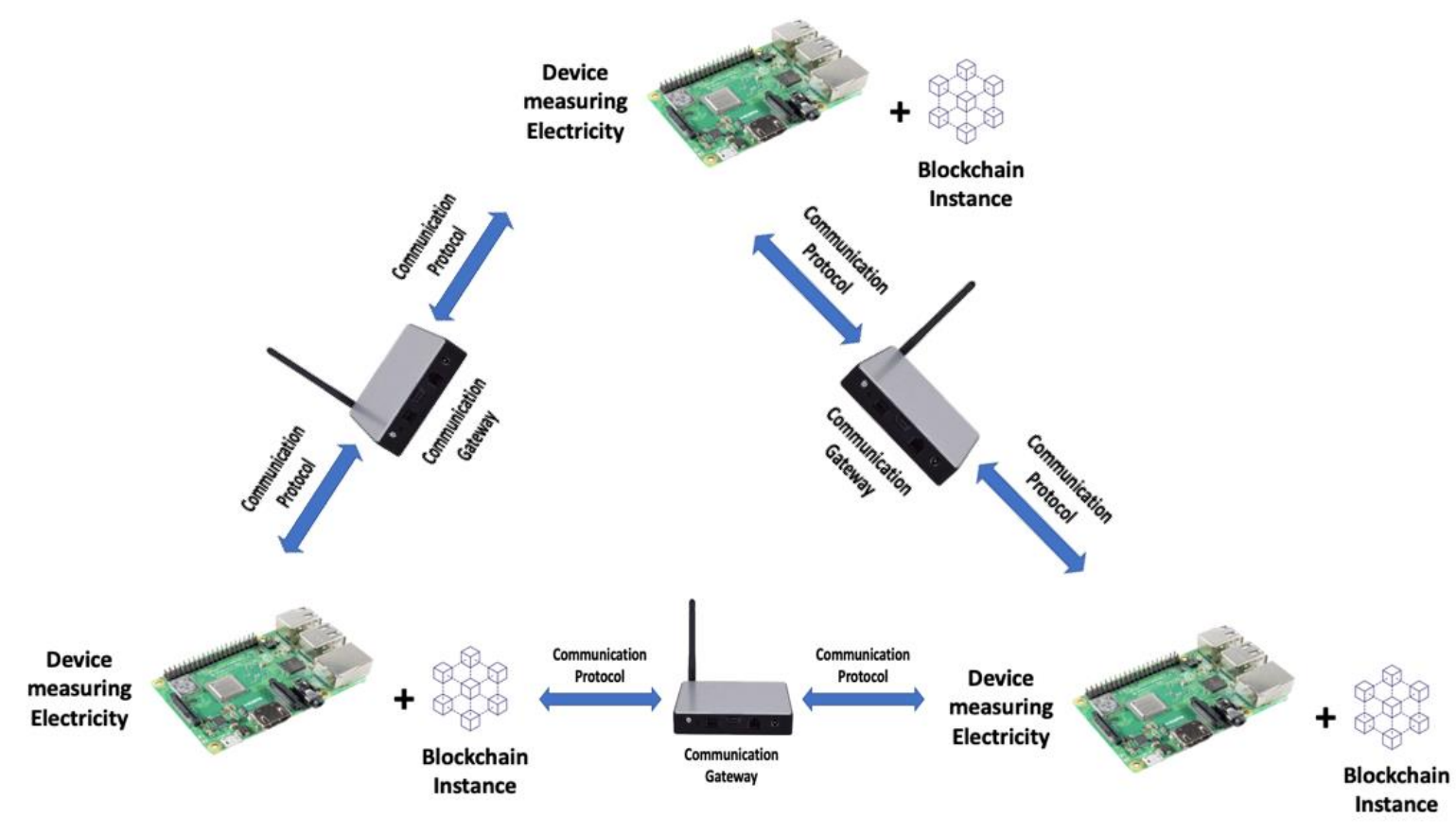

Fig. 8. Proposed architecture

\section{Related Works}

The authors Ahmet Önder Gür et al., in their work "Blockchain Based Metering and Billing System Proposal with Privacy Protection for the Electric Network" [18] propose a Raspberry Pi based Hyperledger blockchain network to implement a smart electricity power consumption meter. Their proposed system aims to solve the cost, privacy and security problems of measurement and billing systems by using a decentralized system. The Raspberry Pi component is used to obtain values from the electricity meter, (simulated for the purpose of experiment) that is connected to the Fabric network. The Hyperledger Fabric, implemented using the SOLO consensus algorithm maintains the blockchain for each user based on the session keys. Additional tools such as CouchDB were chosen to allow complex queries which were used during generating reports.

In their works "Privacy-Preserving and Efficient Aggregation Based on Blockchain for Power Grid Communications in Smart Communities", the authors Zhitao Guan, et al., [19] propose a smart meter to measure and record electricity consumption of the users, in a grid infrastructure. By maintaining separate blockchains for each section of users in the grid, the proposed architecture segregates the network based on the mining capacity of the network users, that is, the rates at which blocks are introduced into the network i.e. more blocks for industrial and lesser for domestic sectors. The proposed model protects the user data using pseudonyms and efficiently mines the network by selecting the miners that have consumption values close to the average of the particular section of the model. The billing is processed on the completion of a fixed cycle after successful validation of the blocks for a period of time.

ISSN: 2582-3825 (online) 
Journal of Electronics and Informatics (2020)

Vol.02/ No. 01

Pages: 49-71

http://www.irojournals.com/iroei/

DOI: https://doi.org/10.36548/jei.2020.1.006

Shafiq Aiman, et al., in their work "Smart Electricity Billing System Using Blockchain Technology" [20] propose a decentralized prepaid wallet energy metering with a conceptual cryptocurrency-based payment system called Wattcoin. The smartphone Wattcoin application retains the cryptocurrencies of the user, which can be used to deploy configurations to the meter such as on/off operations and power saving mode. The smart meter consists of Raspberry Pi ( 2 for purpose of the experiment) computers connected to the energy meter that stores the meter values in the Ethereum chain periodically. The Wattcoin application is also used to view the electricity usage read from the blockchain. Sivaganesan D, et al [36] put forth the internet of things with the BC S. Smys et al [37] performed the "A novel report on architecture, protocols and applications in Internet of Things (IoT)." Raj, J. S et al [38] conducted the "Automation Using Iot in Greenhouse Environment." Shadaram, et al [39] discusses the . "Introduction to the special issue on "Wireless systems: New technologies, resource optimization and security"." Suma, V et al [40] devised the "Security and Privacy Mechanism Using Blockchain"

\section{Techniques and Tools}

The experiment consists of one tool and three hardware components to attain distribution and security. Ethereum Blockchain [6] technology is the tool used and the components used are the Raspberry Pi computer [7], RPICT3 Pi HAT and the SCT-013-000 Alternating Current (AC) Sensor clamp. The Interplanetary File System (IPFS) [8] which is an open-source tool that is capable of attaining distribution, is used to host the El DApp and devoid the network from utilizing dedicated servers for the application. Ethereum is an opensource blockchain platform capable of keeping track of the transactions. The Raspberry Pi computers functions as peers on the network while simultaneously measuring the power usage using the SCT clamp and updating the blockchain for the users.

\subsection{Ethereum Blockchain}

The Ethereum is one of the blockchain technologies available, implemented with the application for the purpose of enhancing the security and decentralization of the application data. A blockchain is a data structure that records data of transactions in a well-defined double linked data structure. The Ethereum is an open source blockchain component that allows developers to utilize its several consensus protocols to implement a secure blockchain backend network. The Ethereum instance is deployed and run on an Ethereum Virtual Machine on which the Ethereum network deploys and executes the smart contracts [3.1.1] during transactions. The Ethereum blockchain used in this application performs based on the Proof-of-Work consensus protocol [3.2.2] while validating the transactions. The El DApp using the blockchain technology utilizes an incentive called Ether, native to the Ethereum platform in order to initiate and validate the transactions, which serves as a pseudo currency to incentivize the miners for validating the transactions.

\subsubsection{Smart Contract}

Similar to a real-life contract, a smart contract is an agreement between the peers using the services, by following and abiding by the rules of the contract. The contract functions as a micro-service for the

ISSN: 2582-3825 (online) 
Journal of Electronics and Informatics (2020)

Vol.02/ No. 01

Pages: 49-71

http://www.irojournals.com/iroei/

DOI: https://doi.org/10.36548/jei.2020.1.006

peers to execute the rules of the contract when a certain criterion is met. But unlike a real-life contract, a smart contract programmed in solidity [9] is capable of executing itself on the Ethereum platform without any intervention by the peers to utilize the services of the network. When any new blockchain is created, the smart contract is deployed at the creation of the chain, such that any block created in the blockchain should have followed the contract before being added to the chain. The setter function of the smart contract that records the electricity usage for a user is shown in listing 1 .

Listing 1: Solidity code of El DApp Smart Contract

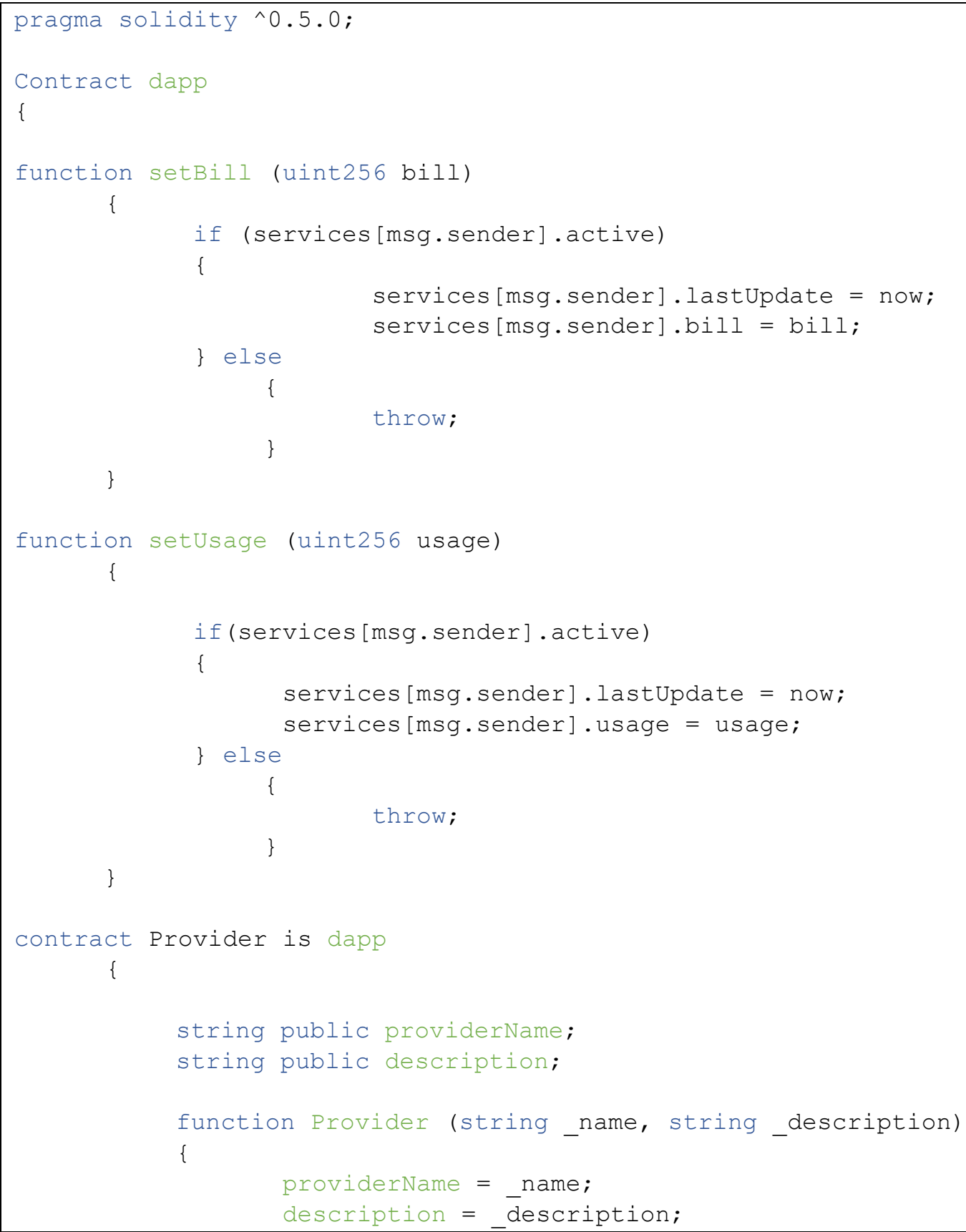


Journal of Electronics and Informatics (2020)

Vol.02/ No. 01

Pages: 49-71

http://www.irojournals.com/iroei/

DOI: https://doi.org/10.36548/jei.2020.1.006

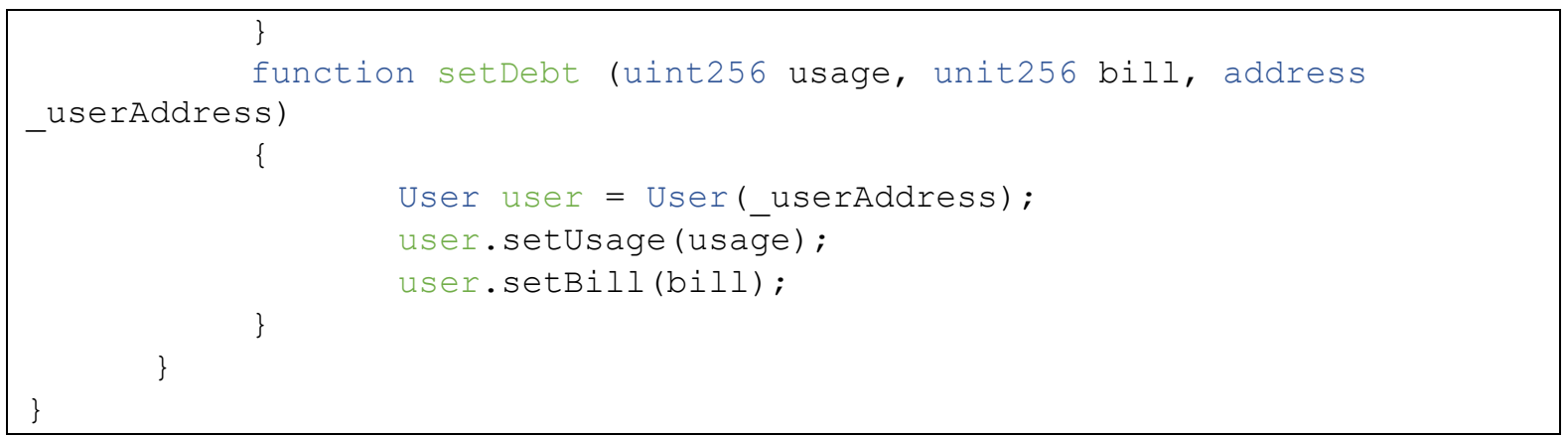

\subsubsection{Proof-of-Work consensus protocol}

In the a blockchain structure, a block is validated before it is added to the blockchain. This mechanism of the consensus is that, at least more than half of the peers on the network must agree to the validity of the block. This is a function that, if the majority of the vote on the block is agreed upon to be valid, then the block is considered valid. The Proof-of-Work protocol awards the peer with the incentive (Ether in this application) based on the computational resource provided in validating the block.

\subsection{Raspberry $P i$}

The Raspberry $\mathrm{Pi}$ is a single board mini-computer designed, developed and manufactured by the Raspberry Pi Foundation in the United Kingdom. This computer has the capability to perform basic tasks with minimum configuration that a fully- fledged low-end computer is capable of performing. Considering the fact that the Internet of Things solutions can be efficiently implemented using a Raspberry Pi, this specific component was chosen in order to test its feasibility of running a private, fully decentralized application. The Raspberry Pi used for this application is the Raspberry Pi version 3 B Plus, having a Broadcom BCM2837B0, Cortex-A53 (ARMv8) 64-bit SoC clocking at 1.4GHz overclocked to $1.54 \mathrm{GHz}, 1 \mathrm{~GB}$ of LPDDR2 SDRAM and $2.4 \mathrm{GHz}$ and $5 \mathrm{GHz}$ IEEE $802.11 \mathrm{~b} / \mathrm{g} / \mathrm{n} / \mathrm{ac}$ wireless LAN, making it suitable for the component to perform as a server for a small numbered cluster. The Raspberry Pi is operated using Raspbian, a Linux based operating system tailored for the Raspberry Pi which has an Ethereum client and the IPFS instance running in its kernel instance, which are also configured to automatically start on the bootup of the Raspberry Pi.

ISSN: 2582-3825 (online) 
Journal of Electronics and Informatics (2020)

Vol.02/ No. 01

Pages: 49-71

http://www.irojournals.com/iroei/

DOI: https://doi.org/10.36548/jei.2020.1.006

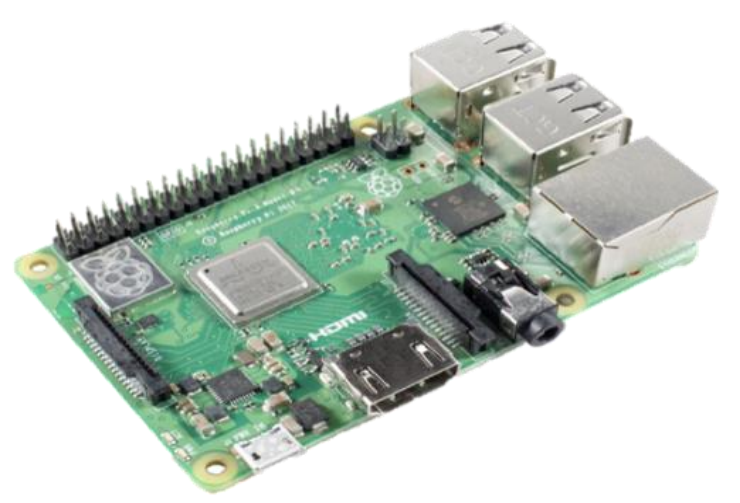

Fig. 9. Raspberry Pi 3 B Plus

\subsection{RPICT3}

The RPICT3 is the version 3 of the RPICT Raspberry Pi hats series for the sensing and measurement of AC currents and temperature. The RPICT3 board connects to the GPIO connectors of the Raspberry $\mathrm{Pi}$ and provides data via the serial port. The Pi hat is configured using the inbuilt Raspbian packages and calibrated to obtain the correct measurement readings from the AC CT clamp sensor.

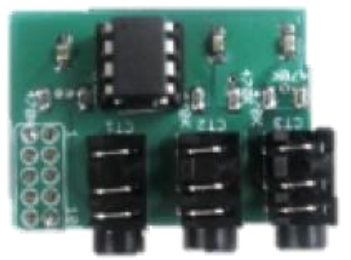

Fig. 10. RPICT3 Raspberry Pi HAT

\subsection{SCT-013-000}

The SCT-013-000 is a 100A Non-Invasive AC Current Sensor Split Core Type Clamp Meter used in the measurement of the AC current to or from the appliance. A magnetic current transformer inside the clamp meter senses the magnetic fluctuations and converts the AC current to an induced AC current on the clamp which is measured by the RPICT3 Pi HAT. The clamp is capable of measuring AC currents between 0 and 100A. The current measured is used in the calculation of power consumed by the appliance. The clamp continuously measures the current, which depends the program utilizing the clamp for readings and the total sum of the current measured per hour is mined to create a block.

ISSN: 2582-3825 (online) 
Journal of Electronics and Informatics (2020)

Vol.02/ No. 01

Pages: 49-71

http://www.irojournals.com/iroei/

DOI: https://doi.org/10.36548/jei.2020.1.006

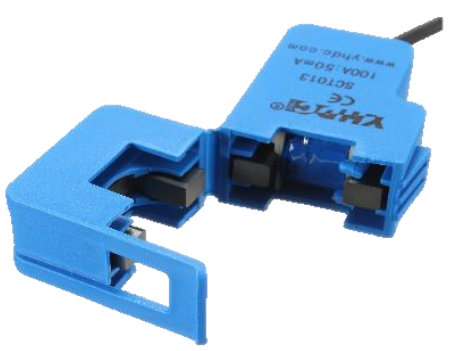

Fig. 11. SCT-013-00 CT Clamp

\section{Experimental Setup and Results}

The experimental setup consists of 4 Raspberry Pi 3 B Plus operated using the Raspbian Operating system with the Ethereum and IPFS configured to start at boot. The front-end of the application and the smart contracts are uploaded to the IPFS to function as a server-less application. The clamp meter program to measure the current is also run as a daemon process, starting at boot on all the peers along with the configured bootstrap file for the IPFS to connect all the nodes on the network. The IPFS hosts the El DApp application enabling the users to view their live electricity usage. The IPFS is made to maintain this state and listen to a dedicated port for all the incoming traffic. The SSH request access, HDMI access and keyboard access are blocked to prevent users from tampering with the Raspberry Pi components. The EVM is made active by realizing the Geth [10] package utilizing the Go Language, developed by Google Inc. The Instance is initiated through a custom Genesis file for the El DApp. The Genesis file is a set of configurations that the blockchain will follow during its creation. The genesis file is given in listing 2 .

Listing 2: El DApp Genesis File

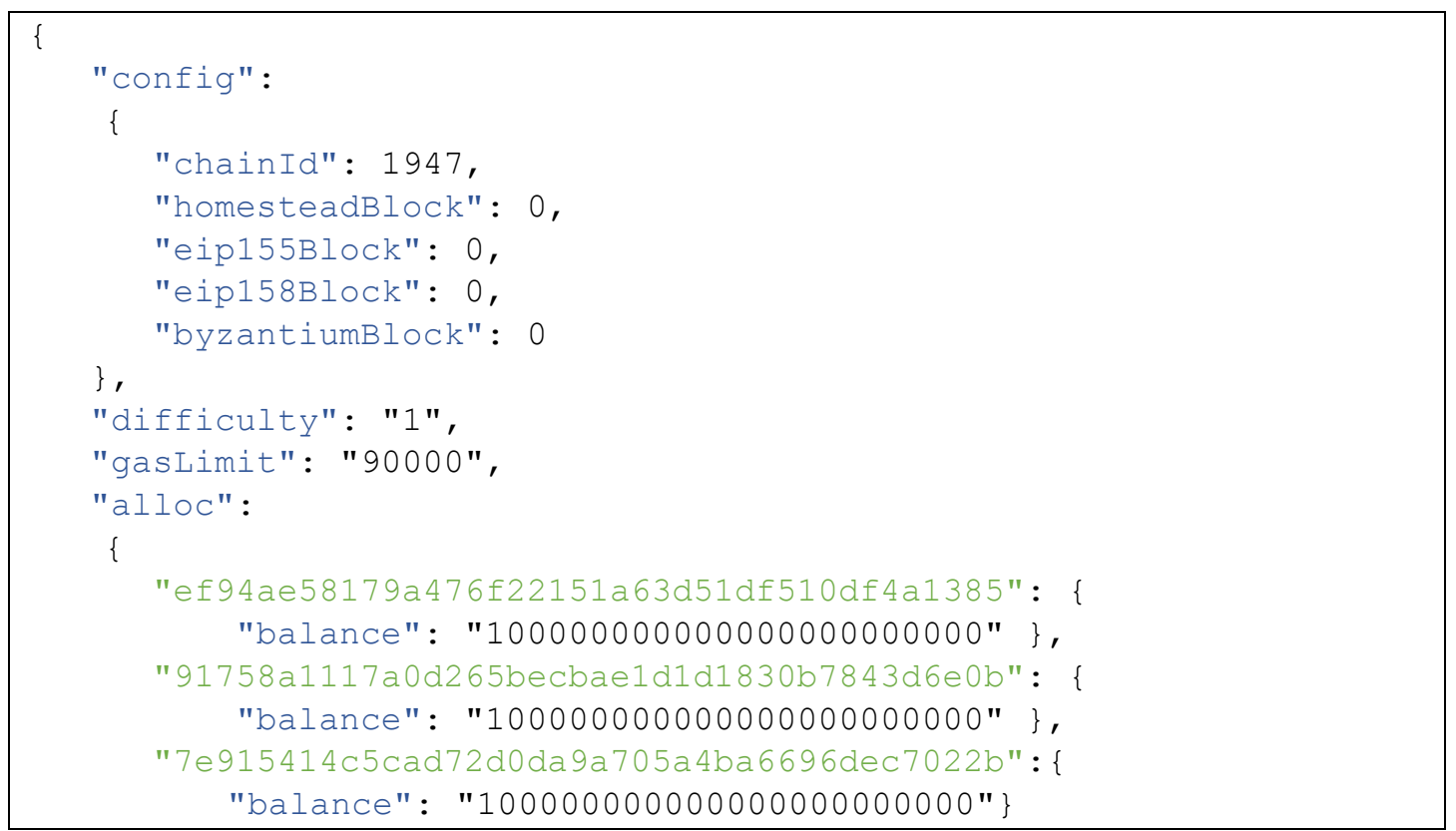

ISSN: 2582-3825 (online) 


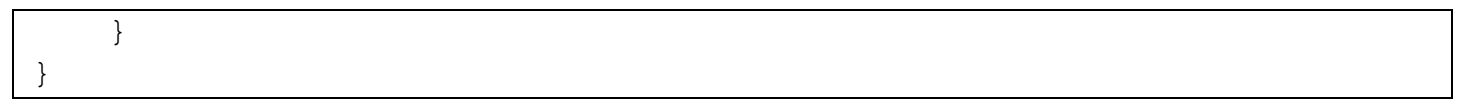

After instantiating the Genesis Block by executing the Genesis file, a new blockchain instance is created, with all the peers connected to this instance from static-nodes.json file in the Geth directory and the accounts in the Genesis files are provided with the mentioned ether balances. Since the mining time increases gradually during the mining process of blocks in the blockchain with the increase in chain and network size, the consensus time must be kept at constant, to avoid memory overload on the Raspberry Pi. This is achieved by editing the consensus file of the Raspberry Pi Ethereum instance and setting the time (in milliseconds) of the difficulty to a low value. Listing 3 shows the consensus file for the Ethereum instance of a Raspberry Pi.

Listing 3: Difficulty function in Consensus file

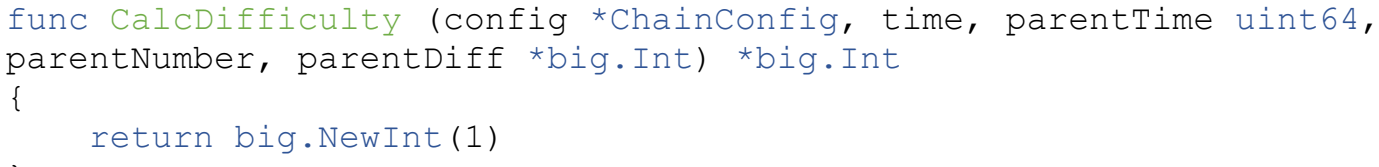

The Geth instance is initiated on bootup with the auto DAG generation disabled to avoid the Raspberry Pi running out of memory. The JavaScript console, native to the Ethereum platform is a tool, utilized in obtaining details of the current Geth blockchain instance. The peers connected to the current network can be found from the console, using the command admin.peers. The number of peers connected to a particular peer can be found from the console, using the net.peerCount command.

When a new transaction is initiated and must be validate i.e. mined, the Ethereum network requires the peer to provide a certain amount of incentive to validate the transaction. This incentive is called Gas, which is a fraction of Ether. It is measured in Gwei. Gas is the measure of the incentive, based amount of computational power required to process a block, whereas Ether is the standard currency measure in the Ethereum platform.

$$
1 \text { Gwei }=10^{-9} \text { Ether }
$$

Gwei is the standard used in the Genesis configuration file [Listing 2]. A fraction of this Ether (Gas) is used, depending on the Gas price set by the Genesis File, for every transaction throughout the network whenever the smart contract is executed. The ether balance for any peer account can be found in the JavaScript console using the function eth.getBalance(eth.coinbase). These ethers amount shown in the account do not represent the main network ether and can only be transferred to any account on the private network. 
Journal of Electronics and Informatics (2020)

Vol.02/ No. 01

Pages: 49-71

http://www.irojournals.com/iroei/

DOI: https://doi.org/10.36548/jei.2020.1.006

The smart contract for the blockchain is instantiated using the Truffle Blockchain Framework [11] after the creation and initiation of the Genesis block. This process is done only once and the blockchain follows the current smart contract throughout the execution cycle. Once the contract is executed on the blockchain, the block returns an Application Binary Interface (ABI) and the address of the contract on the blockchain. The two factors are utilized using the Web3.py package [12] to execute the contract directly and avoid recompilation. This process is given in the Fig. 12.

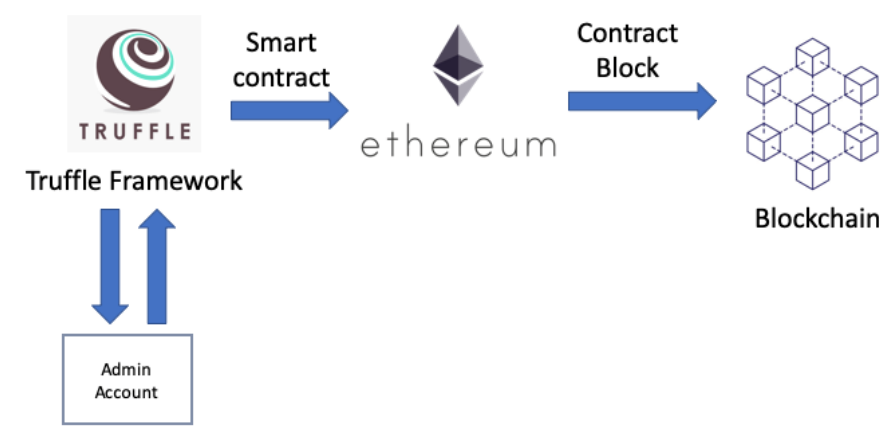

Fig. 12. Smart Contract initiation using Truffle

Once the smart contract is deployed, Metamask [13], an Ethereum bridge tool that manages accounts of the configured Ethereum RPC, is capable of interacting with the Ethereum RPC instance and is utilized to initiate transactions.

The CT clamp, used to measure the electric current of an appliance, is connected to a SAMSUNG AR18KV5HBTR 1.5 Ton Inverter Split Air Conditioner without a stabilizing unit for the purpose of this experiment. The clamp measures the current at a range between $6.23 \mathrm{~A}$ to $6.91 \mathrm{~A}$ in the operating environment. The power utilized by the appliance can be found from the obtained current value using equation 1.

$$
P=V I \text { watt }
$$

According to the Tamil Nadu Electricity Board, the voltage in the domestic sector ranges between $220 \mathrm{~V}$ and $240 \mathrm{~V}$. Using the equation 1 , the calculated power consumed, ranges between $1370.6 \mathrm{~W}$ and $1520.2 \mathrm{~W}$, which is in accordance with the appliance specification of $1520 \mathrm{~W}$ input power. 
Journal of Electronics and Informatics (2020)

Vol.02/ No. 01

Pages: 49-71

http://www.irojournals.com/iroei/

DOI: https://doi.org/10.36548/jei.2020.1.006

The python program that calculates the power consumption and the amount to be paid for the power units, directly communicates with the Ethereum instance using python Web3 to execute the contract without any intermediate APIs. The Raspberry Pi components are configured to mine the value of the total power consumed every hour, calculating power in $\mathrm{kWh}$, also known as units of power or units. The total power consumed every hour is stored in the blockchain and a list that stores the total power consumption per day in the contract, is updated at the end of an Indian Standard 24-hour period, which is shown as a usage graph in the application [Fig. 15,16]. The Ethereum interaction process with the energy meter program is given in listing 4.

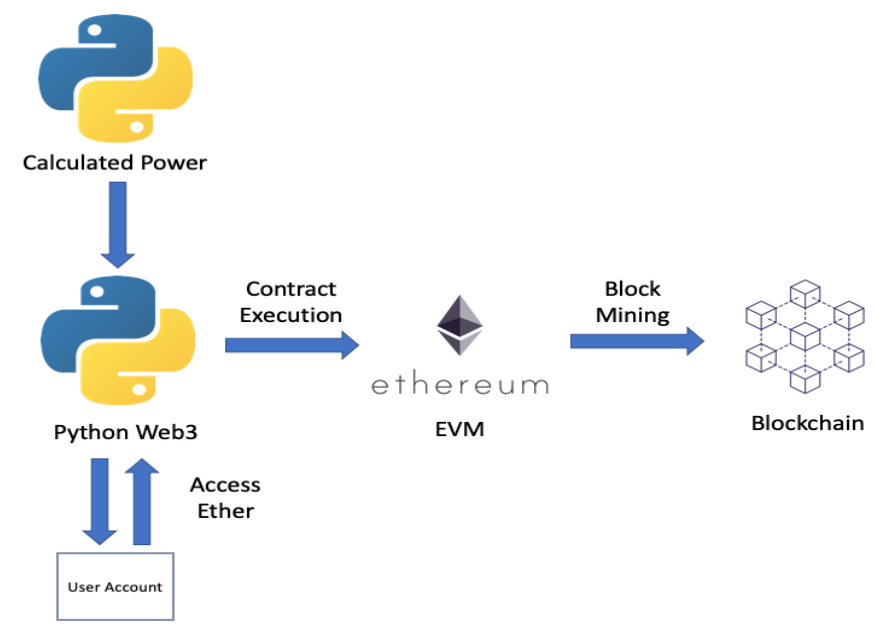

Fig. 13. Block creation process

Listing 4: Block creator (Python Web3) program.

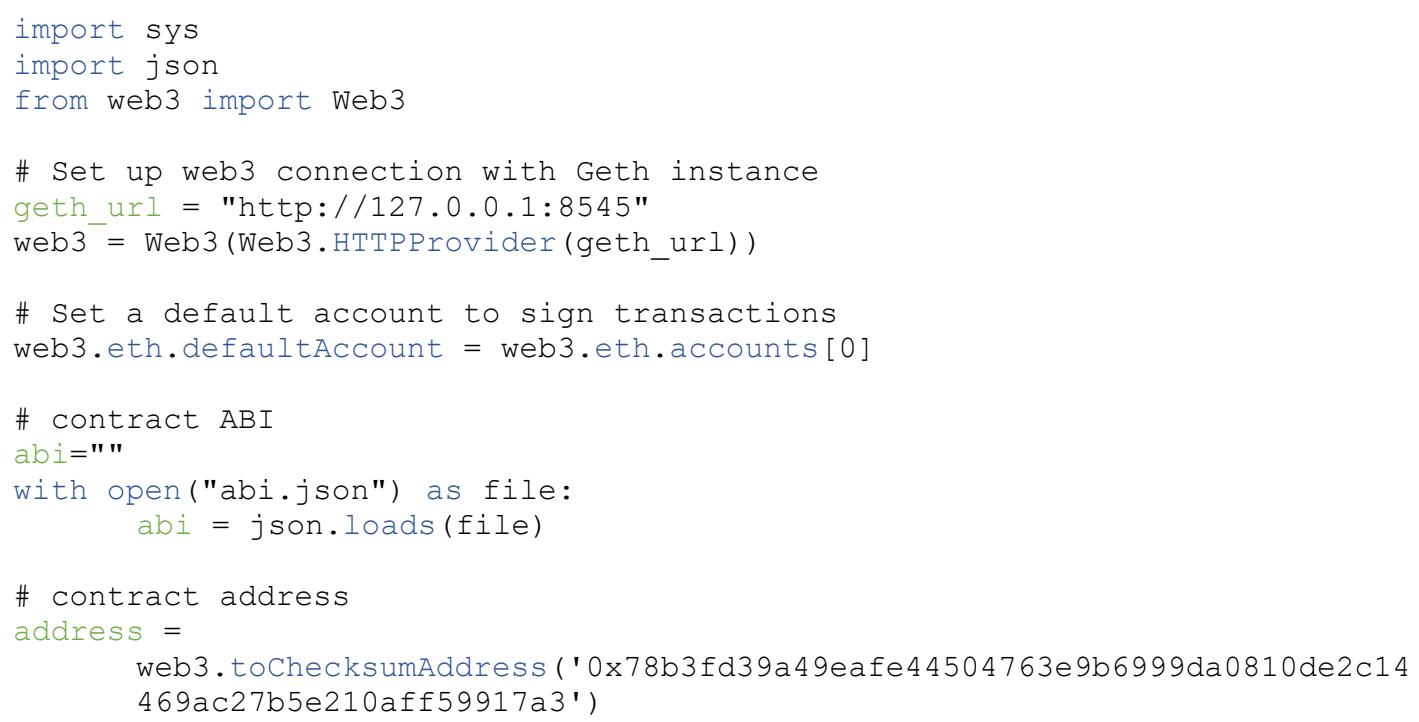


Journal of Electronics and Informatics (2020)

Vol.02/ No. 01

Pages: 49-71

http://www.irojournals.com/iroei/

DOI: https://doi.org/10.36548/jei.2020.1.006

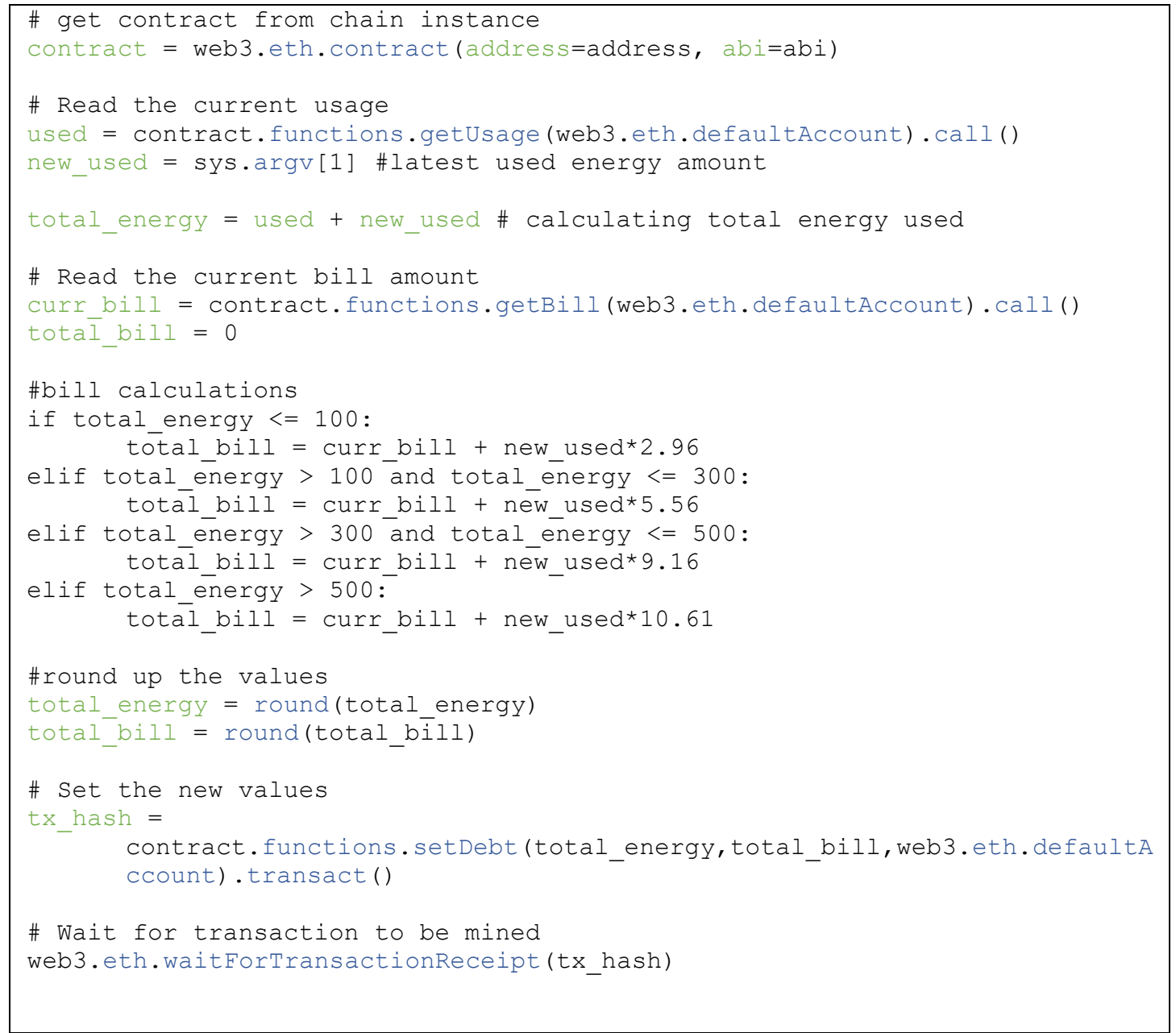

The back-end algorithm of the El DApp and the Ethereum instance is given in Table. 1.

Table. 1. Pseudo code for Back-End Algorithm

Pseudo code for Back-End Algorithm

\section{Back-End Algorithm}

Begin

Instantiate Ethereum with smart contract.

Repeat

if (Time elapsed==1 hour and IST day not completed)

Deploy contract with calculated power and amount to pay

Mine Block

if (mine $==$ successful)

Add block to blockchain

if (Time elapsed==1 hour and IST day completed)

Deploy contract with calculated power and amount to pay

Update usage list in contract

Mine Block

if (mine $==$ successful)

Add block to blockchain

if (El DApp request==GET values)

ISSN: 2582-3825 (online) 
Journal of Electronics and Informatics (2020)

Vol.02/ No. 01

Pages: 49-71

http://www.irojournals.com/iroei/

DOI: https://doi.org/10.36548/jei.2020.1.006

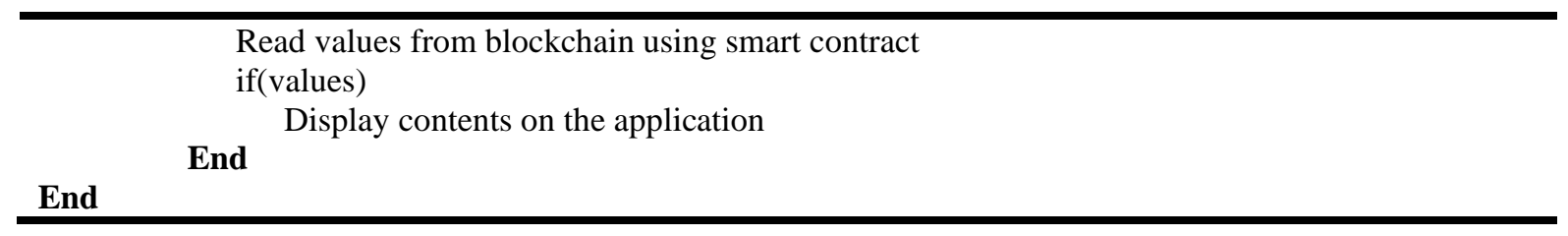

The user logins in to the account with which the Raspberry Pi of the user mines the power usage, using the Metamask UI and the private key given for that particular account. The user is prompted to approve the access for the account used by Metamask initially, after connecting the Geth RPC [Fig. 14]. This allows the El DApp to utilize the contract read function and show measurements statistics. Once the page is loaded, the index page [Fig. 15,16] shows the power consumptions statistics based on the IST time at which the user views their account.

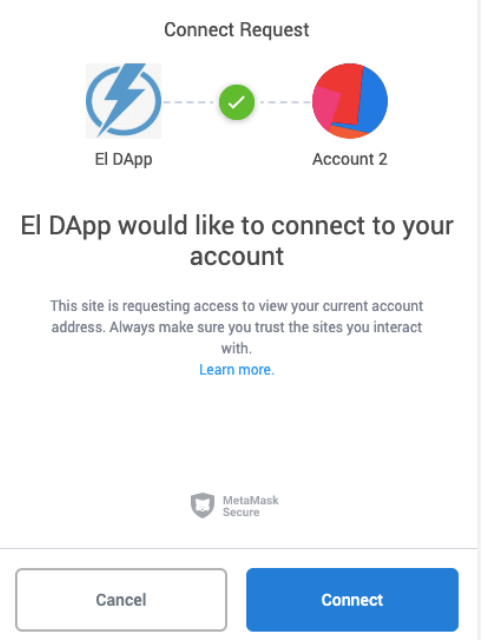

Fig. 14. Metamask Account access prompt 
Journal of Electronics and Informatics (2020)

Vol.02/ No. 01

Pages: 49-71

http://www.irojournals.com/iroei/

DOI: https://doi.org/10.36548/jei.2020.1.006

\section{EDApp}

YOUR ACCOUNT

OxEf94aE58179a476f22151a63D51DF510dF4a1385

TOTAL POWER CONSUMPTION (from 1/Jan/2020 to 12/Jan/2020) 112 UNITS

BILL AMOUNT (from 1/Jan/2020 to 12/Jan/2020) ₹ 188.00

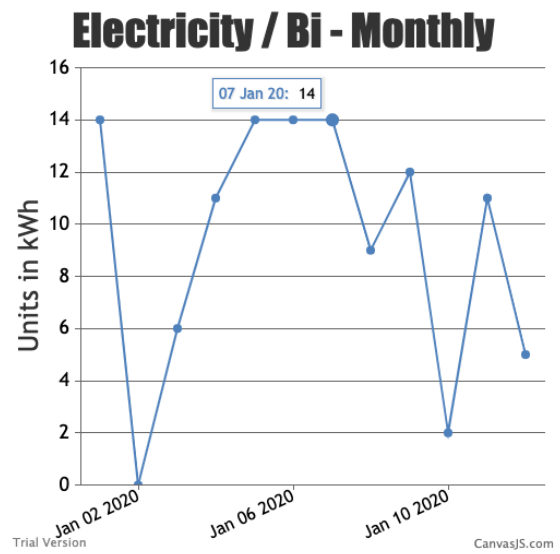

Fig. 15. El DApp Air Conditioner power usage statistics from 1/Jan/2020 to 12/Jan/2020

\section{BDApp}

YOUR ACCOUNT

OXEf94aE58179a476f22151a63D51DF510dF4a1385

TOTAL POWER CONSUMPTION (from 1/Jan/2020 to 31/Jan/2020) 245 UNITS

BILL AMOUNT (from 1/Jan/2020 to 31/Jan/2020) ₹ 565.00

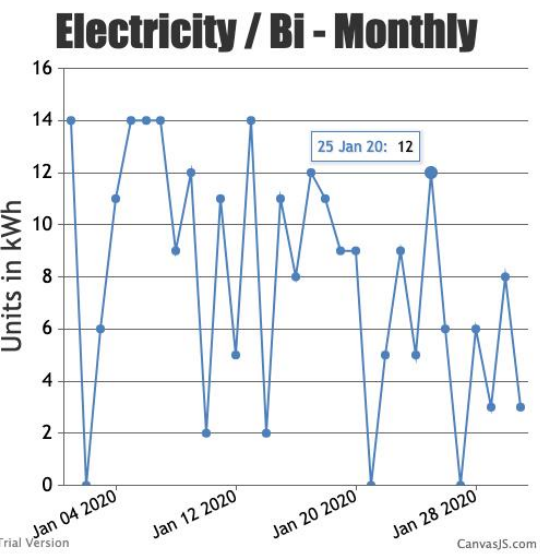

Fig. 16. El DApp Air Conditioner power usage statistics from 1/Jan/2020 to 12/Jan/2020

The El DApp utilizes Metamask to access and show the power consumption statistics for a particular account on the Ethereum network. The Metamask serves as an account manager for the El DApp. This mechanism is shown in figure 17. 
Journal of Electronics and Informatics (2020)

Vol.02/ No. 01

Pages: 49-71

http://www.irojournals.com/iroei/

DOI: https://doi.org/10.36548/jei.2020.1.006

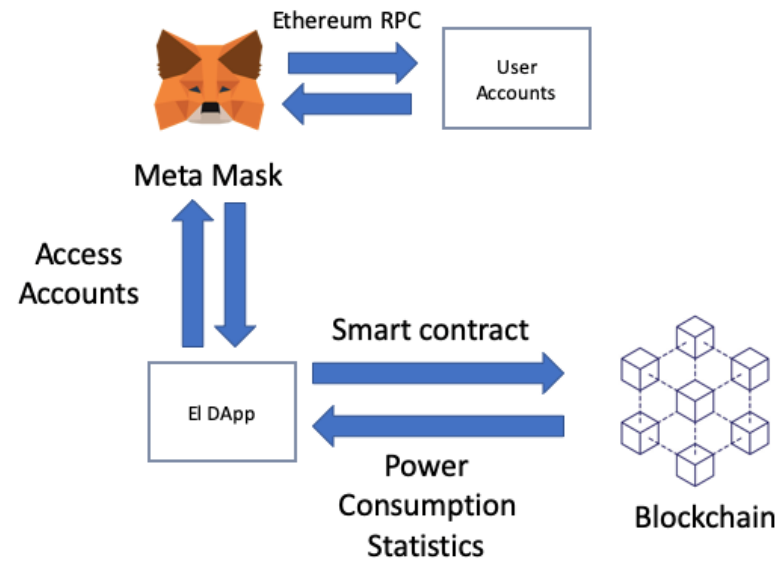

Fig. 17. Account management in El DApp

The blockchain state is changed based on the consensus that majority of the network peers must agree with an occurrence of an event. Hence any peer trying to tamper with the blockchain must alter at least $51 \%$ of the peers to cause liable alteration in security, which is exponentially increased in difficulty as the size of the network increases. Deeming this network to be secure and tamper-proof. The sequence of operations of the El DApp working mechanism is given in figure 18.

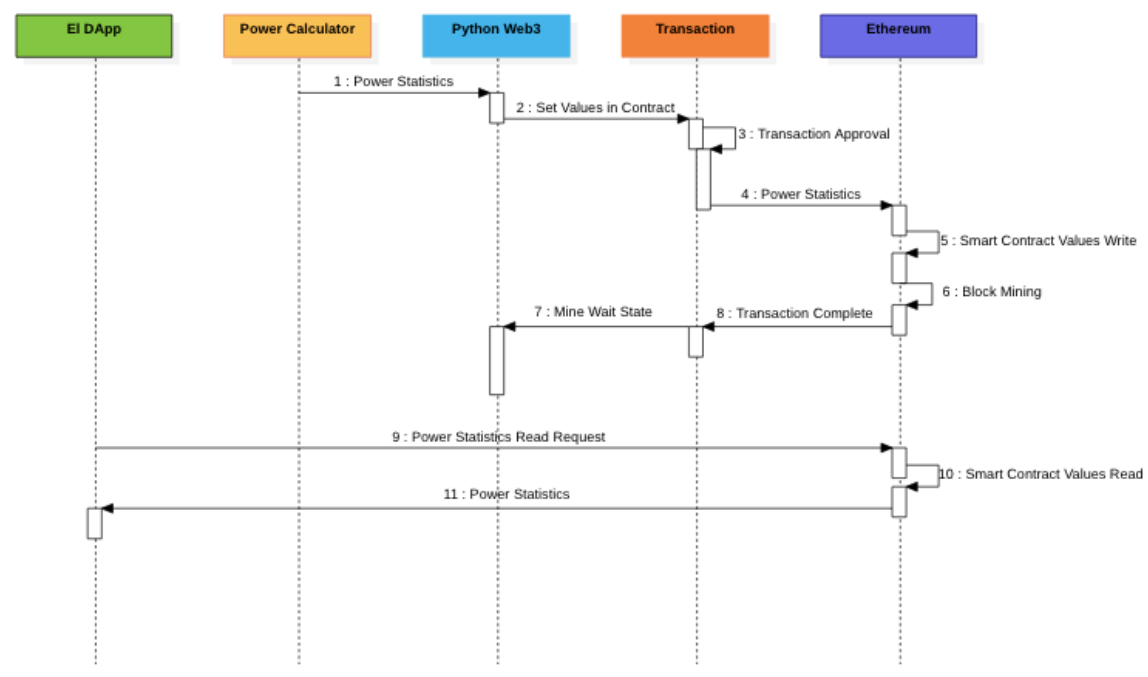

Fig. 18. El DApp sequence of operations

During the validation of the blocks for a transaction, as the size of the network increases the computational time and the computational difficulty decreases. Since the difficulty to mine the block remains constant for the network chain, the mining pool increases the computational utilization thereby optimal block validation. Table 2. shows the CPU usage and the time taken to mine a block by the Raspberry-Pi peers. 
Journal of Electronics and Informatics (2020)

Vol.02/ No. 01

Pages: 49-71

http://www.irojournals.com/iroei/

DOI: https://doi.org/10.36548/jei.2020.1.006

Table. 2. Mining CPU usage and Time taken per Block

\begin{tabular}{ccc}
\hline $\begin{array}{c}\text { No. of Peers } \\
\text { (Raspberry Pi) }\end{array}$ & $\begin{array}{c}\text { CPU Usage \% } \\
\text { (Geth) }\end{array}$ & $\begin{array}{c}\text { Avg. Time/Block } \\
\text { (seconds) }\end{array}$ \\
\hline 2 & 12 & 9 \\
3 & 7 & 5 \\
4 & 4.2 & 3 \\
\hline
\end{tabular}

The mining operations can be improved significantly by utilizing ASIC machines for the network and an adaptive difficulty should implemented for the same to improve security.

\subsection{Results}

\subsubsection{Security Analysis}

The blockchain technology is considered to be one of the most secure methods of storing data in a decentralized as opposed to the centralized method of data storage. Some of the security benefits that follow this solution are as follows.

- The data of a user, stored in the blockchain is retained even if the component of the user gets damaged, as the distribution of data eliminates the problem of single point of failure.

- Any mal-practice performed by the user is annulled, as the data in the blockchain cannot be changed once set. Even if the user tries to tamper with their component, the Raspberry-Pi is configured to block all physical connections and repeated non-administrative SSH connections to configure the mining process.

- The blockchain technology is reliable, as the chain follows the consensus protocol before altering the state of the chain, thereby making the system devoid of inconsistency.

\subsubsection{Cost-Benefit Analysis}

The smart meter architecture costs significantly high with the smart meter component itself costing between the range of ₹ 4,000 and ₹ 10,000 by the manufacturer. Along with the component additional functionalities such as database maintenance, security programs cost would increase the initial cost.

On the other hand, the proposed model costs approximately ₹ 5,000, which is a database and a security solution inclusive method that has comparatively lower maintenance cost than the traditional client server architecture. 
Journal of Electronics and Informatics (2020)

Vol.02/ No. 01

Pages: 49-71

http://www.irojournals.com/iroei/

DOI: https://doi.org/10.36548/jei.2020.1.006

\section{Performance Analysis}

Although the performance of the configured proposed model has a performance advantage over the traditional database solution, when deployed as a real-life solution with adaptive difficulty and more powerful machines to mine the network, the performance degrades with respect to the difficulty of the blockchain. The time taken to update the chain by adding a block is given by equation 2 .

$$
\text { time }(t)=\text { difficulty }(D) \times 2^{32} / \text { hash rate }(H)
$$

This degradation in performance is a trade-off for the security and cost-benefit.

\section{Conclusion}

In this paper, the deployment and the mechanics of El DApp, a decentralized Electricity power consumption tracking application using blockchain, is demonstrated utilizing a Raspberry-Pi network. This article provides an insight on the following functionalities of the El DApp.

- The feasibility of a decentralized application to track power consumptions.

- The security and automation benefits of the application such as, a fool-proof energy meter and the ease in tracking power consumption.

- The feasibility of a Raspberry Pi network to maintain a blockchain network state.

This solution also prevents and concurrently assists in voiding the claims of users on swindled electricity bills and usage records on the user by the respective electricity providers.

\subsection{Limitations}

Although the Raspberry Pi nodes are able to host and maintain the blockchain network and only mine the network in intervals of 1 block an hour, one of the most fundamental requirements of the network is that the peers must be online and synchronous with the network which requires a dedicated network connectivity. The solution also does not address the physical security, such damage to the RaspberryPi components.

\subsection{Future Works}

ISSN: 2582-3825 (online) 
Journal of Electronics and Informatics (2020)

Vol.02/ No. 01

Pages: 49-71

http://www.irojournals.com/iroei/

DOI: https://doi.org/10.36548/jei.2020.1.006

This article proposes a private decentralized application utilizing the Ethereum blockchain. Consequently, currency based on the regulated system of exchange is immoral to use on this private network as the only mode of payment. Hence, this solution can be improved by offering an ICO for the network with regulated exchange currency with which the users will be able pay for the electricity consumption via the application.

\section{References}

[1] https://data.worldbank.org/indicator/EG.USE.ELEC.KH.PC?end=2018\&most_recent_val ue_desc $=$ false $\&$ start $=1960 \&$ type $=$ shaded $\&$ view $=$ chart $\&$ year $=2013$.

[2] https://www.statista.com/statistics/471264/iot-number-of-connected-devices-worldwide.

[3] https://www.statista.com/statistics/543089/iot-security-spending-worldwide.

[4] https://www.statista.com/statistics/781804/smart-electricity-meter-market-size-in-the-us.

[5] https://www.statista.com/statistics/878748/worldwide-production-phase-blockchaintechnology-industry.

[6] https://ethereum.org.

[7] https://www.raspberrypi.org/products/raspberry-pi-3-model-b-plus.

[8] https://ipfs.io.

[9] https://solidity.readthedocs.io/en/v0.5.0/resources.html.

[10] https://geth.ethereum.org.

[11] https://www.trufflesuite.com.

[12] https://web3py.readthedocs.io/en/stable.

[13] https://metamask.io.

[14] https://eandt.theiet.org/content/articles/2019/03/youtube-videos-showing-how-to-tamperwith-energy-meters.

[15] https://www.theguardian.com/technology/2016/dec/29/smart-electricity-metersdangerously-insecure-hackers.

[16] Mamula O, Mejzrova L \& Vodrazka J (2018) Failure Analysis of Current and Future Electricity Meters and their Components in Relation to the Costs of Ownership. doi: 10.15598/aeee.v16i2.2547

[17] Biswas, K., \& Muthukkumarasamy, V. (2016). Securing Smart Cities Using Blockchain Technology. 2016 IEEE 18th International Conference on High Performance Computing and Communications; IEEE 14th International Conference on Smart City; IEEE 2nd International Conference on Data Science and Systems (HPCC/SmartCity/DSS). doi:10.1109/hpcc-smartcity-dss.2016.0198

[18] Gur, A. O., Oksuzer, S., \& Karaarslan, E. (2019). Blockchain Based Metering and Billing System Proposal with Privacy Protection for the Electric Network. 2019 7th International Istanbul Smart Grids and Cities Congress and Fair (ICSG). doi:10.1109/sgcf.2019.8782375.

[19] Guan, Z., Si, G., Zhang, X., Wu, L., Guizani, N., Du, X., \& Ma, Y. (2018). PrivacyPreserving and Efficient Aggregation Based on Blockchain for Power Grid Communications in Smart Communities. IEEE Communications Magazine, 56(7), 8288. doi:10.1109/mcom.2018.1700401.Aiman S, Hassan S, Habbal A, Rosli A \& Shabli A. 
Journal of Electronics and Informatics (2020)

Vol.02/ No. 01

Pages: 49-71

http://www.irojournals.com/iroei/

DOI: https://doi.org/10.36548/jei.2020.1.006

Smart Electricity Billing System Using Blockchain Technology. ISSN: 2289-8131 Vol. 10 No. 2-4.

[20] Huh, S., Cho, S., \& Kim, S. (2017). Managing IoT devices using blockchain platform. 2017 19th International Conference on Advanced Communication Technology (ICACT). doi:10.23919/icact.2017.7890132.

[21] Panarello, A.; Tapas, N.; Merlino, G.; Longo, F.; Puliafito, A. Blockchain and IoT Integration: A Systematic Survey. Sensors 2018, 18, 2575.

[22] Zhang, Y., \& Wen, J. (2016). The IoT electric business model: Using blockchain technology for the internet of things. Peer-to-Peer Networking and Applications, 10(4), 983-994. doi:10.1007/s12083-016-0456-1.

[23] Rao, A. R., \& Clarke, D. (2019). Perspectives on emerging directions in using IoT devices in blockchain applications. Internet of Things, 100079. doi:10.1016/j.iot.2019.100079.

[24] Lazaroiu, C., \& Roscia, M. (2017). Smart district through IoT and Blockchain. 2017 IEEE 6th International Conference on Renewable Energy Research and Applications (ICRERA). doi:10.1109/icrera.2017.8191102.

[25] Zhang, Y., \& Wen, J. (2015). An IoT electric business model based on the protocol of bitcoin. 2015 18th International Conference on Intelligence in Next Generation Networks. doi:10.1109/icin.2015.7073830.

[26] Andoni, M., Robu, V., Flynn, D., Abram, S., Geach, D., Jenkins, D., Peacock, A. (2019). Blockchain technology in the energy sector: A systematic review of challenges and opportunities. Renewable and Sustainable Energy Reviews, 100, 143-174. doi:10.1016/j.rser.2018.10.014.

[27] Li, Z., Kang, J., Yu, R., Ye, D., Deng, Q., \& Zhang, Y. (2017). Consortium Blockchain for Secure Energy Trading in Industrial Internet of Things. IEEE Transactions on Industrial Informatics, 1-1. doi:10.1109/tii.2017.2786307.

[28] Buth, M. C. (Annemarie), Wieczorek, A. J. (Anna), \& Verbong, G. P. J. (Geert). (2019). The promise of peer-to-peer trading? The potential impact of blockchain on the actor configuration in the Dutch electricity system. Energy Research \& Social Science, 53, 194-205. doi:10.1016/j.erss.2019.02.021.

[29] Andersen M, Kolb J, Chen K, Fierro G, Culler D \& Popa R, 2017 Technical Report No. UCB/EECS-2017-234. WAVE: A Decentralized Authorization System for IoT via Blockchain Smart Contracts.

[30] Cao, Y. (2019). Energy Internet blockchain technology. The Energy Internet, 45-64. doi:10.1016/b978-0-08-102207-8.00003-5.

[31] Qusay F. Hassan - Internet of Things A to Z: Technologies and Applications, Chapter - 9.

[32] Kamal, M., \& Tariq, M. (2019). Light-weight Security and Blockchain Based Provenance for Advanced Metering Infrastructure. IEEE Access, 1-1. doi:10.1109/access.2019.2925787.

[33] Rottondi, C., \& Verticale, G. (2017). A Privacy-Friendly Gaming Framework in Smart Electricity and Water Grids. IEEE Access, 5, 14221-14233. doi:10.1109/access.2017.2727552.

[34] Lombardi, F., Aniello, L., De Angelis, S., Margheri, A., \& Sassone, V. (2018). A Blockchain-based Infrastructure for Reliable and Cost-effective IoT-aided Smart Grids.

ISSN: 2582-3825 (online) 
Journal of Electronics and Informatics (2020)

Vol.02/ No. 01

Pages: 49-71

http://www.irojournals.com/iroei/

DOI: https://doi.org/10.36548/jei.2020.1.006

Living in the Internet of Things: Cybersecurity of the IoT - 2018. doi:10.1049/cp.2018.0042.

[35] G. Wood, "Ethereum: A secure decentralised generalised transaction ledger," Ethereum Project Yellow Paper, vol. 151, 2014.

[36] Sivaganesan, D. (2019). Block Chain Enabled Internet of Things. Journal of Information Technology, 1(01), 1-8.

[37] Kumar, R. Praveen, and S. Smys. "A novel report on architecture, protocols and applications in Internet of Things (IoT)." In 2018 2nd International Conference on Inventive Systems and Control (ICISC), pp. 1156-1161. IEEE, 2018.

[38] Raj, J. S., \& Ananthi, J. V. (2019). Automation Using Iot in Greenhouse Environment. Journal of Information Technology, 1(01), 38-47.

[39] Shadaram, Mehdi, S. Smys, and Sherali Zeadally. "Introduction to the special issue on "Wireless systems: New technologies, resource optimization and security"." Computers and Electrical Engineering 2, no. 40 (2014): 289-290.

[40] Suma, V. (2019). Security And Privacy Mechanism Using Blockchain. Journal of Ubiquitous Computing and Communication Technologies (UCCT), 1(01), 45-54. 